Research Article

\title{
Network Pharmacology Identifies the Mechanisms of Sang-Xing-Zhi-Ke-Fang against Pharyngitis
}

\author{
Yinhe Deng, ${ }^{1}$ Quanjiang Li, ${ }^{1}$ Menglin Li, ${ }^{1}$ Tiantian Han, ${ }^{1}$ Guixian $\mathrm{Li}^{1}{ }^{1}$ and Qiong Liu $\mathbb{D}^{2}$ \\ ${ }^{1}$ College of First Clinical Medical, Guangzhou University of Chinese Medicine, Guangzhou 51000, China \\ ${ }^{2}$ International Medical Department, The First Affiliated Hospital of Guangzhou University of Chinese Medicine, \\ Guangzhou 51000, China \\ Correspondence should be addressed to Qiong Liu; 1423254686@qq.com
}

Received 1 April 2020; Revised 1 September 2020; Accepted 9 September 2020; Published 12 October 2020

Academic Editor: Rômulo Dias Novaes

Copyright (C) 2020 Yinhe Deng et al. This is an open access article distributed under the Creative Commons Attribution License, which permits unrestricted use, distribution, and reproduction in any medium, provided the original work is properly cited.

Background. Sang-Xing-Zhi-Ke-Fang (SXZKF) demonstrates good therapeutic effect against pharyngitis. Nevertheless, the pharmacological mechanism underlying its effectiveness is still unclear. Objective. To investigate the underlying mechanisms of SXZKF against pharyngitis using network pharmacology method. Methods. Bioactive ingredients of SXZKF were collected and screened using published literature and two public databases. Using four public databases, the overlapping genes between these bioactive compound-related and pharyngitis-related genes were identified by Venn diagram. Protein-protein interaction (PPI) was obtained using "Search Tool for the Retrieval of Interacting Genes (STRING)" database. "Database for Annotation, Visualization, and Integrated Discovery ver. 6.8 (DAVID 6.8)" was used to perform Kyoto Encyclopedia of Genes and Genomes (KEGG) pathway enrichment analysis to explore the molecular mechanisms of SXZKF against pharyngitis. Finally, Cytoscape 3.7.2 software was used to construct and visualize the networks. Result. A total of 102 bioactive compounds were identified. Among them, 886 compounds-related and 6258 pharyngitis-related genes were identified, including 387 overlapping genes. Sixty-three core targets were obtained, including ALB, PPAR $\gamma$, MAPK3, EGF, and PTGS2. Signaling pathways closely related to mechanisms of SXZKF for pharyngitis were identified, including serotonergic synapse, VEGF signaling pathway, Fc epsilon RI signaling pathway, Ras signaling pathway, MAPK signaling pathway, and influenza A. Conclusion. This is the first identification of in-depth study of SXZKF against pharyngitis using network pharmacology. This new evidence could be informative in providing new support on the clinical effects of SXZKF on pharyngitis and for the development of personalized medicine for pharyngitis.

\section{Introduction}

Pharyngitis, a collective term for inflammation caused by various microorganisms in the pharynx, still has a high incidence even with the rapid development of modern medicine [1]. In China, acute pharyngitis is mainly treated with antiviral drugs, antibiotics or traditional Chinese medicine (TCM) [2,3]. TCM plays an increasingly important role in the treatment of chronic pharyngitis, for the etiology of chronic pharyngitis is complex, and many studies have shown that TCM shows significant effect on this disease $[4,5]$.

Sang-Xing-Zhi-Ke-Fang is a concoction based on SangXing-Tang, which is originated form Wen Bing Tiao
BiannBia that had been widely used for more than 200 years in China. SXZKF has been patented in China (patent number: 201910302604.4) in 2019 and the oral lozenge based on it is being developed. SXZKF is composed of Mulberry Leaf, Armeniacae Amarum Semen, Radix Glehniae, Radix Rehmanniae Praeparata, Loguat Leaf, Fritillary Bulb, Eriobotrya japonica Thunb, Ligusticum chuanxiong hort, Earthworm Lumbricus, Mentha haplocalyx Briq, and Exocarpium Citrus Grandis. Although we have previously verified that SXZKF was effective against pharyngitis [6], the active ingredients and molecular mechanisms of SXZKF in the treatment of pharyngitis remains unknown.

Network pharmacology is a new discipline based on the theory of systems biology, analyzing the network of 
biological systems, and selecting specific signal nodes for multitarget drug molecular design [7]. Based on the ideas and methodologies of network pharmacology, this study aimed to obtain the main active ingredients of SXZKF, screened out the core targets and main biological pathways, and explored the molecular mechanisms of SXZKF in the treatment of pharyngitis.

\section{Material and Methods}

2.1. Screening of Bioactive Compounds of SXZKF. Information of the bioactive compounds in SXZKF was obtained from Traditional Chinese Medicine Systems Pharmacology Database and Analysis Platform (TCMSP, http://lsp.nwu.edu.cn/tcmsp.php) and Bioinformatics Analysis Tool for Molecular mechanism of Traditional Chinese Medicine (BATMAN-TCM, http://bionet.ncpsb. org/batman-tcm/) [8]. The active ingredients of SXZKF were filtered using the following criteria: (i) oral bioavailability $(\mathrm{OB}) \geq 30 \%$ and (ii) drug-likeness (DL) $\geq 0.18[9,10]$. Other ingredients were obtained through literature analysis $[11,12]$.

\subsection{Screening of Target Genes of Bioactive Compounds and} Pharyngitis. Target genes of bioactive compounds of SXZKF were obtained from TCMSP, and the Universal Protein Database (UniProt, https://www.uniprot.org/) was then used to remove nonhuman target gene names. To ensure the reliability of prediction, only target genes with "Reviewed/ Swiss-Prot" rots "Human" in UniProt would be selected $[13,14]$. The targets related to pharyngitis were obtained from Comparative Toxicogenomics Database (CTD, http:// ctdbase.org/) [15] and The Human Gene Database (GeneCards, http://www.genecards.org/). By searching the keyword "pharyngitis," target genes of pharyngitis were identified.

\subsection{Establishment of Herbs-Compounds-Targets (H-C-T)} Network. The overlapping target genes relating to both the bioactive compounds and pharyngitis were identified and visualized by Venn diagrams (http://bioinformatics.psb. ugent.be/webtools/Venn/). Then, Cytoscape 3.7.2 software was used to visualize and analyze the network of interactions between overlapping genes, bioactive compounds, and herbs; we named it the $\mathrm{H}-\mathrm{C}-\mathrm{T}$ network.

2.4. Analysis of Network Topological Features. STRING database (https://string-db.org/) was used to obtain the protein-protein interactions (PPI) by uploading 387 overlapping targets between active compounds and pharyngitis. Species was limited to "Homo sapiens" with a confidence score $>0.4$. Cytoscape 3.7.2 was used to construct the network of PPI, and then the topological features of the network were analyzed to screen out the core targets that play a crucial part in the PPI network. The degree centrality (DC), betweenness centrality (BC), and closeness centrality (CC) were analyzed for each node using the plugin cytoNCA in Cytoscape 3.7.2 [16]. Preliminary screening was carried out for nodes with a DC equal or larger than the median of two. Finally, the nodes with BC and $\mathrm{CC}$, both larger than the median of two, were identified as the core targets [17].

2.5. Pathway Analyses and Construction of CompoundsTargets-Pathways (C-T-P) Network. Pathway enrichment analysis for overlapping genes was performed using DAVID ver. 6.8 (https://david.ncifcrf.gov/) with the "Homo sapiens" setting. Generally, the results of KEGG pathway enrichment were considered to have statistically significant and necessary functional mechanisms of pharyngitis when $P<0.05$. To ensure the accuracy of this study, we set the screening criteria as $P<0.01$. Bubble chart of the concerned KEGG pathways was plotted using the OmicShare tools (https:// www.omicshare.com/tools). Finally, Cytoscape 3.7.2 was used to establish and visualize the network of interactions between compounds, targets, and signaling pathways that are closely related to pharyngitis. The complete workflow of the analysis is given in Figure 1.

\section{Results}

3.1. Bioactive Compounds of SXZKF. Ninety-three bioactive compounds were identified from TCMSP and BATMANTCM, while another nine bioactive compounds were identified through literature survey. Among these, 29 compounds were identified from Mulberry Leaf, 19 compounds from Semen Armeniacae Amarum Semen, 8 compounds from Radix Glehniae, 2 compounds from Radix Rehmanniae Praeparata, 18 compounds from Loguat Leaf, 10 compounds from Mentha haplocalyx Briq, 7 compounds from Fritillary Bulb,7 compounds from Ligusticum chuanxiong hort, 10 compounds from Exocarpium Citrus Grandis, and 9 compounds from Earthworm Lumbricus (Table S1).

3.2. Target Genes of SXZKF and Pharyngitis. A total of 886 targets genes (Table S2) of 63 bioactive compounds were retrieved from TCMSP and screened by UniProt, and no genes were related to another 39 compounds. On the other hand, 6258 targets related to pharyngitis were obtained (5150 targets from CTD, 1803 targets from GeneCards; among them 695 duplicate targets), and the information is listed in Table S3. As shown in Figure 2, the Venn diagram showed that 387 overlapping target genes were identified by matching the SXZKF compounds-related target genes with CTD and GeneCards pharyngitis-related target genes.

3.3. Analysis of H-C-T Network. Based on the Venn diagram and the results of TCMSP retrieval, we can obtain that the 387 potential targets were linked to 19 compounds of SXZKF against pharyngitis, as shown in Table S4. To get a better standing of the interactions between herbs, compounds, and 


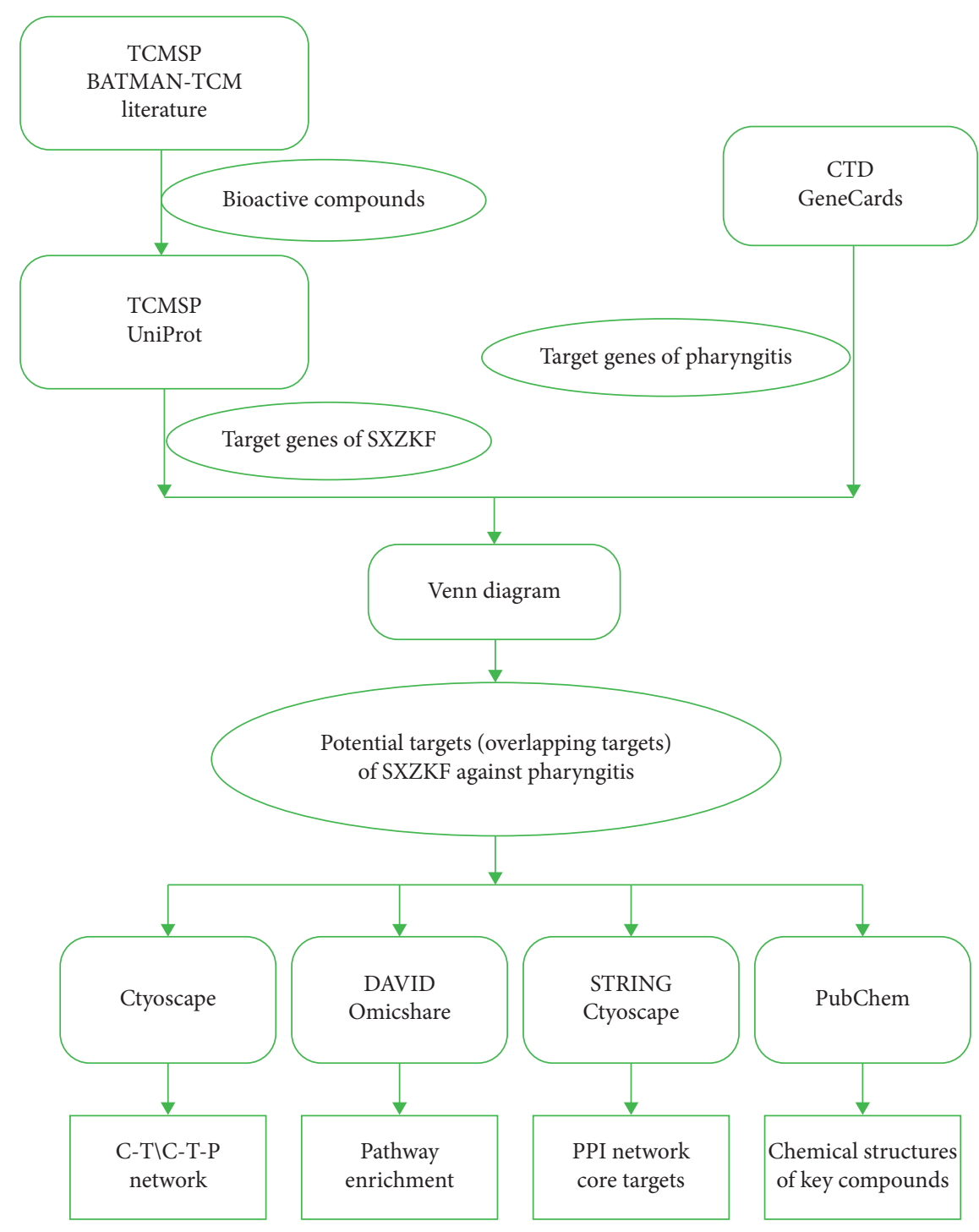

FIgURE 1: Workflow of this study.

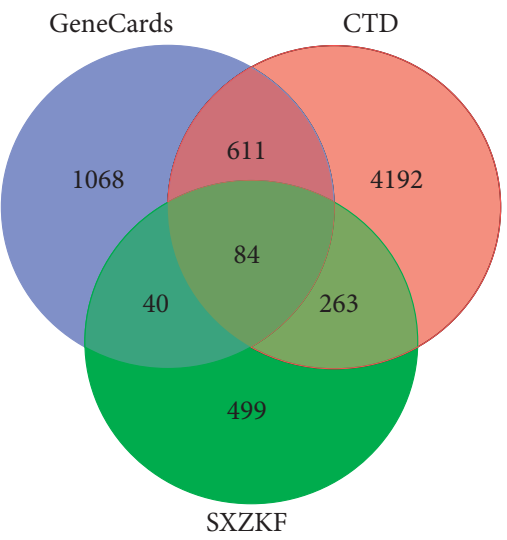

FIGURE 2: 387 overlapping genes between SXZKF compounds-related targets and pharyngitis-related targets from GeneCards and CTD.

target genes, a visual network with 415 nodes (including 9 herbs, 19 compounds, and 387 targets) and 546 edges was established by Cytoscape as shown in Figure 3.
3.4. PPI Network and Core Targets. We used the plugin cytoNCA in the Cytoscape 3.7.2 to calculate the degree centrality (DC), betweenness centrality (BC) and closeness centrality (CC) for each node in PPI network, the results are shown in Table S5. According to these values, the targets with DC, BC, and CC larger than the median of two were used as core targets for subsequent analysis (as shown in Figure 4). As displayed in Table 1, 63 core targets were finally obtained.

3.5. Pathway Enrichment Analysis. 387 potential targets were mapped to a total of 109 signaling pathways using DAVID, 28 of which were identified as $P<0.01$ (as shown in Table S6). Top 20 KEGG pathways' enrichment analysis is shown in Figure 5 and Table 2.

3.6. Analysis of C-T-P Network. After reviewing the published literature, we narrowed down to 6 of the top 20 signaling pathways, which are closely related to pharyngitis. 


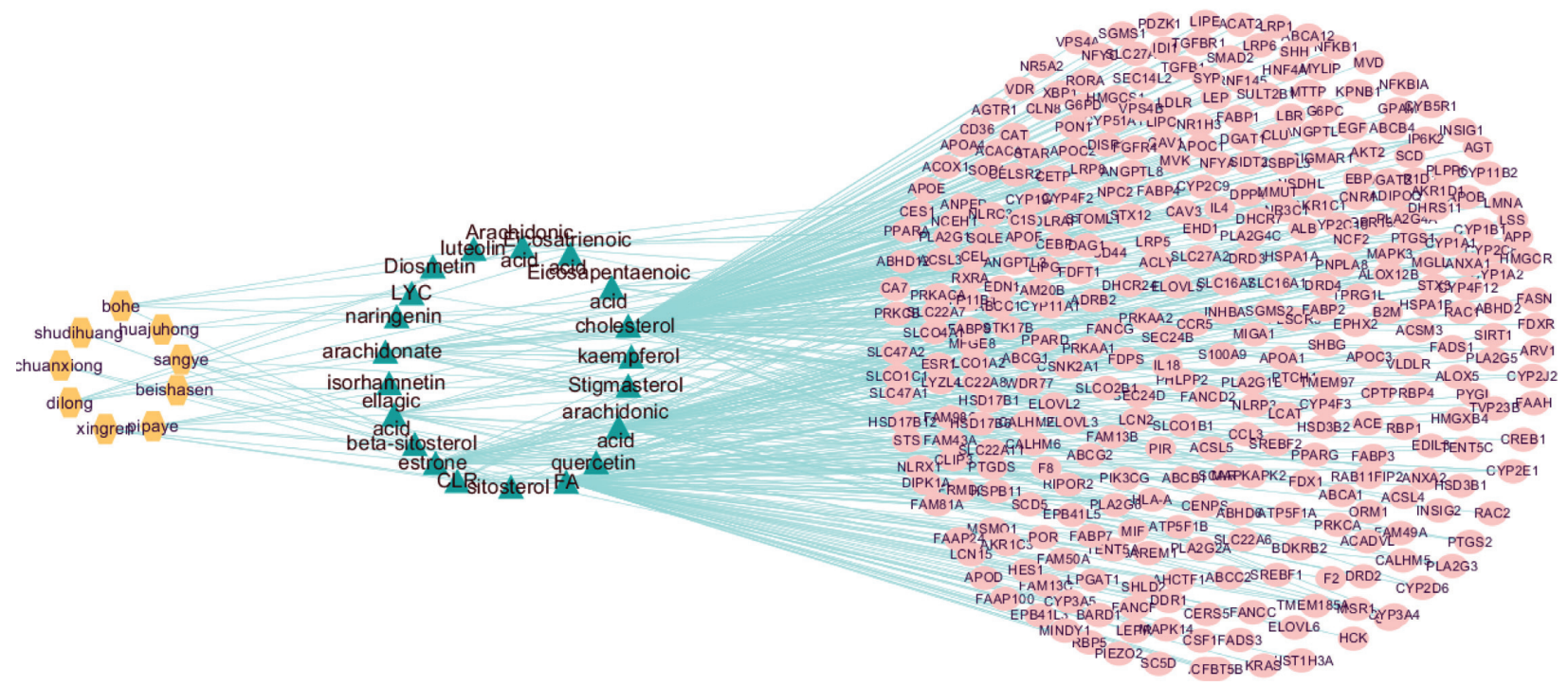

FIGURE 3: H-C-T network. Yellow nodes represent the herbs of SXZKF, green nodes represent the bioactive compounds of SXZKF, and pink nodes represent the potential targets of SXZKF against pharyngitis.

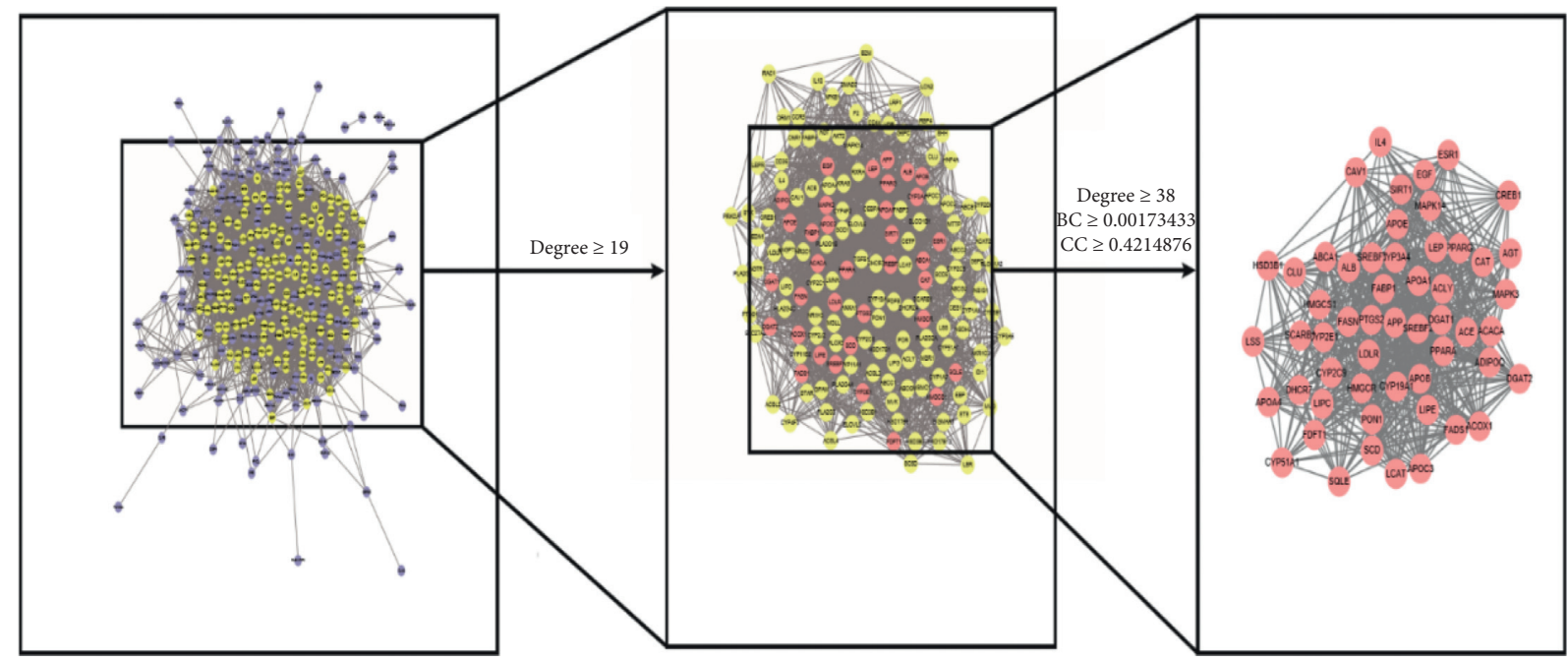

FIGURE 4: Screening of core targets by analyzing topological features of the PPI network. Red nodes represent the core targets.

These includes serotonergic synapse, VEGF signaling pathway, Fc epsilon RI signaling pathway, Ras signaling pathway, MAPK signaling pathway, and influenza A. Cytoscape 3.7.2 was used to construct the network of "compounds-key pathways-targets," consisting of 59 nodes (including 10 compounds, 6 pathways, and 43 targets) and 158 edges, as demonstrated in Figure 6.

\section{Discussion}

As seen in Figures 3 and 6, SXZKF has effects on multiple components, multiple targets, and multiple pathways, which also indicates that TCM plays a synergistic role in the treatment of diseases. Many studies have shown that acute pharyngitis is caused by viral or bacterial infection, and the overlapping pathogenic bacteria include group $\mathrm{A}$ beta-hemolytic streptococcus (the most overlapping) as well as groups $C$ and $G$ streptococcus. In addition, influenza A virus, influenza B virus, rhinovirus, and respiratory syncytial virus are important factors causing pharyngitis [18]. Although the etiology of chronic pharyngitis is complex, bacterial infection is now affirmed as the most important cause, followed by noninfectious factors, such as obstructive sleep apnea hypopnea syndrome, occupational exposure, laryngeal reflux, and allergic diseases. Noninfectious factors combined with microbial infection can induce resistance in the disease progression [19]. Meanwhile, many research have shown that a variety of cytokines are related to the occurrence and development of both acute and chronic pharyngitis. These cytokines include inflammatory factor, tumor necrosis factor (TNF), and arachidonic acid and its 
TABLE 1: Detailed information of 63 core targets.

\begin{tabular}{|c|c|c|c|c|}
\hline NO. & Target name & Degree centrality & Betweenness centrality & Closeness centrality \\
\hline 1 & ALB & 132 & 0.10194051 & 0.57963875 \\
\hline 2 & SREBF1 & 101 & 0.04648343 & 0.54058193 \\
\hline 3 & APOB & 88 & 0.02798912 & 0.52373887 \\
\hline 4 & APOE & 87 & 0.03066411 & 0.52765321 \\
\hline 5 & $\operatorname{PPAR} \gamma$ & 84 & 0.02496889 & 0.53082707 \\
\hline 6 & MAPK3 & 82 & 0.03803816 & 0.52923538 \\
\hline 7 & HMGCR & 79 & 0.02839721 & 0.51911765 \\
\hline 8 & EGF & 77 & 0.0326961 & 0.51683748 \\
\hline 9 & LDLR & 74 & 0.01786479 & 0.5 \\
\hline 10 & FASN & 74 & 0.01620772 & 0.502849 \\
\hline 11 & PPARA & 74 & 0.01758367 & 0.5130814 \\
\hline 12 & PTGS2 & 73 & 0.03636725 & 0.50573066 \\
\hline 13 & SREBF2 & 72 & 0.02642683 & 0.4862259 \\
\hline 14 & APP & 68 & 0.02026671 & 0.50500715 \\
\hline 15 & LEP & 67 & 0.01366234 & 0.50864553 \\
\hline 16 & ABCA1 & 66 & 0.02050839 & 0.49509116 \\
\hline 17 & SCD & 66 & 0.01940855 & 0.48891967 \\
\hline 18 & APOA1 & 65 & 0.0104694 & 0.48891967 \\
\hline 19 & CAT & 59 & 0.0231604 & 0.50070922 \\
\hline 20 & ADIPOQ & 59 & 0.01236963 & 0.49788434 \\
\hline 21 & CYP3A4 & 59 & 0.01670894 & 0.48356164 \\
\hline 22 & ESR1 & 58 & 0.0240653 & 0.49509116 \\
\hline 23 & DGAT1 & 54 & 0.00895186 & 0.47446237 \\
\hline 24 & CYP2B6 & 53 & 0.01261496 & 0.48422497 \\
\hline 25 & APOC3 & 51 & 0.00521994 & 0.45844156 \\
\hline 26 & ACACA & 51 & 0.0040295 & 0.47638327 \\
\hline 27 & CREB1 & 51 & 0.01442992 & 0.49164345 \\
\hline 28 & SIRT1 & 50 & 0.01736748 & 0.49301676 \\
\hline 29 & MAPK14 & 48 & 0.00877424 & 0.47702703 \\
\hline 30 & FABP1 & 48 & 0.01145724 & 0.46693122 \\
\hline 31 & CAV1 & 48 & 0.01458166 & 0.48092643 \\
\hline 32 & SQLE & 47 & 0.00778386 & 0.44853875 \\
\hline 33 & AGT & 47 & 0.00760543 & 0.47510094 \\
\hline 34 & CLU & 47 & 0.00773464 & 0.45844156 \\
\hline 35 & LIPE & 47 & 0.00794991 & 0.47638327 \\
\hline 36 & FADS1 & 47 & 0.00692009 & 0.45607235 \\
\hline 37 & LIPC & 46 & 0.00999712 & 0.44968153 \\
\hline 38 & CYP2E1 & 46 & 0.01342474 & 0.4738255 \\
\hline 39 & IL4 & 45 & 0.00870765 & 0.47192513 \\
\hline 40 & ACOX1 & 45 & 0.0035187 & 0.45784695 \\
\hline 41 & HMGCS1 & 44 & 0.00325402 & 0.44853875 \\
\hline 42 & ACLY & 44 & 0.01071917 & 0.46083551 \\
\hline 43 & CYP19A1 & 44 & 0.01030837 & 0.47255689 \\
\hline 44 & CYP2C9 & 44 & 0.00700349 & 0.46754967 \\
\hline 45 & SCARB1 & 44 & 0.00915057 & 0.47003995 \\
\hline 46 & FDFT1 & 43 & 0.00425437 & 0.45140665 \\
\hline 47 & DGAT2 & 43 & 0.00166345 & 0.44291092 \\
\hline 48 & APOA4 & 43 & 0.00578781 & 0.45314506 \\
\hline 49 & CD44 & 43 & 0.01055526 & 0.46816976 \\
\hline 50 & CYP51A1 & 41 & 0.00425467 & 0.44180225 \\
\hline 51 & HSD3B1 & 41 & 0.00690686 & 0.44180225 \\
\hline 52 & DHCR7 & 41 & 0.00378824 & 0.42891859 \\
\hline 53 & PON1 & 41 & 0.00478681 & 0.46693122 \\
\hline 54 & LSS & 40 & 0.00646986 & 0.44570707 \\
\hline 55 & $\mathrm{ACE}$ & 40 & 0.00407366 & 0.47255689 \\
\hline 56 & ABCG2 & 40 & 0.02206365 & 0.46816976 \\
\hline 57 & F2 & 39 & 0.00736964 & 0.47319035 \\
\hline 58 & KRAS & 39 & 0.00749875 & 0.46143791 \\
\hline 59 & LCAT & 39 & 0.00395196 & 0.46204188 \\
\hline 60 & NR3C1 & 39 & 0.00692536 & 0.47003995 \\
\hline 61 & FABP4 & 38 & 0.0045635 & 0.44796954 \\
\hline 62 & ABCB1 & 38 & 0.01106088 & 0.46693122 \\
\hline 63 & HNF4A & 38 & 0.01122196 & 0.47574124 \\
\hline
\end{tabular}




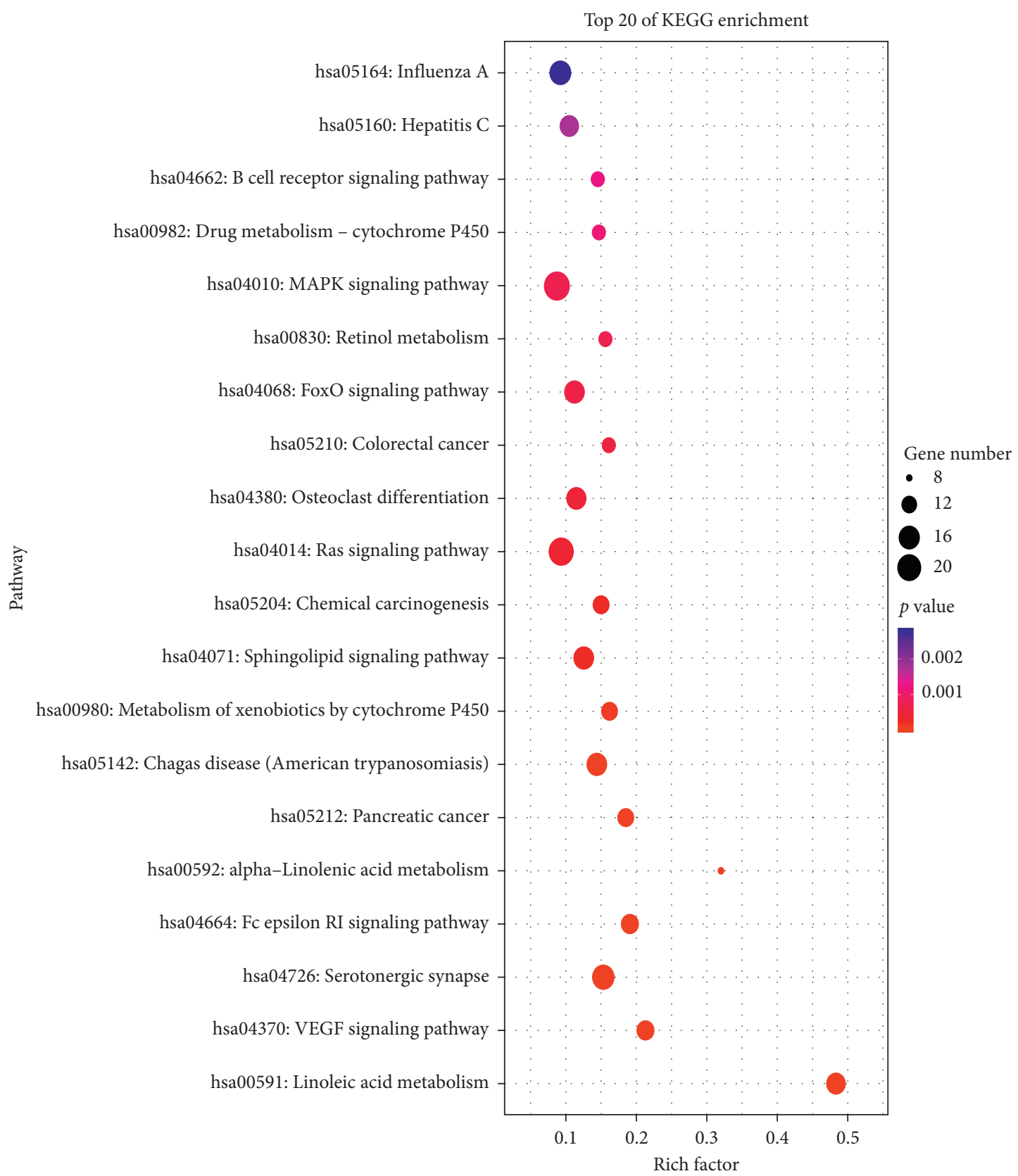

FIGURE 5: Bubble chart of top 20 signaling pathways linked to SXZKF against pharyngitis. Bubble size represented the number of genes enriched in this pathway, color depth represented the $P$ value, and rich factor represented the ratio of the enriched targets in the pathway to the total number of targets in the pathway.

cyclooxygenase metabolites, serotonin (5-HT, also known as serotonin), interleukins (IL-1, IL-2, IL-4, IL-6, IL-10, etc.), epidermal growth factor (EGF), and so on [20].

In our current study, a total of 102 bioactive ingredients were identified in SXZKF. The main active ingredients are arachidonic acid, quercetin, kaempferol, eicosapentaenoic acid, and luteolin, the compounds with higher degree value of node in the H-C-T and C-T-P network, whose chemical structures were shown in Figure 7. Among, the main active ingredients identified, metabolites of arachidonic acid (AA) contribute to inflammation as well as to resolving inflammation. Zhang et al. study demonstrated that AA can directly bind to TLR4 coreceptor, myeloid differentiation factor 2 (MD2), and prevent saturated fatty acids from activating TLR4 proinflammatory signaling pathway. AA can also reduce lipopolysaccharide- (LPS-) induced inflammation in macrophages and septic death in mice through binding to MD2 [21]. Eicosapentaenoic acid (EPA) plays an anti-inflammatory role in the body, which can interfere with the PPAR $\alpha-\mathrm{I} \kappa \mathrm{B}-\mathrm{NF}-\kappa \mathrm{B}$ signaling pathway in inflammatory cells to inhibit the inflammatory response due to the strong anti-inflammatory effects of resolvin and protectin [22]. The proinflammatory and anti-inflammatory effects of AA and EPA intercoordinate to prevent excessive 


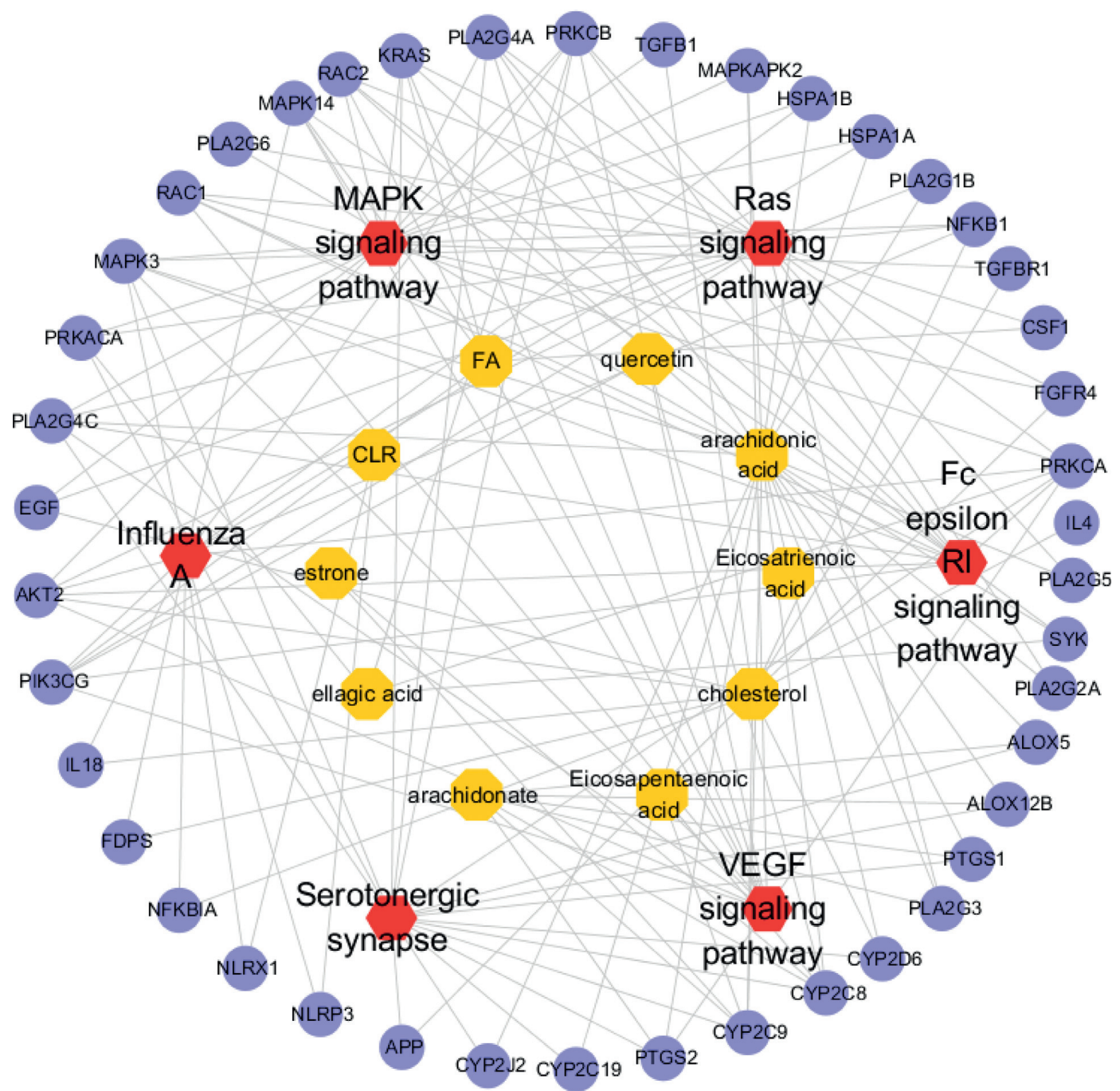

Figure 6: C-T-P network. Yellow nodes represent the bioactive compounds, red nodes represent the key pathways, and purple nodes represent the target genes.<smiles>CCCCC/C=C\C/C=C\C/C=C\C/C=C\CCCC(=O)O</smiles>

(a)<smiles>O=c1c(O)c(-c2ccc(O)c(O)c2)oc2cc(O)cc(O)c12</smiles>

(b)<smiles>O=c1c(O)c(-c2ccc(O)cc2)oc2cc(O)cc(O)c12</smiles>

(c)

Figure 7: Continued. 
<smiles>CC/C=C\C/C=C\C/C=C\C/C=C\C/C=C\CCCC(=O)O</smiles>

(d)<smiles>O=c1cc(-c2ccc(O)c(O)c2)oc2cc(O)cc(O)c12</smiles>

(e)

Figure 7: Chemical structures of key compounds. (a) Arachidonic acid. (b) Quercetin. (c) Kaempferol. (d) Eicosapentaenoic acid. (e) Luteolin.

TABLE 2: Functions of 387 target genes based on KEGG pathway analysis.

\begin{tabular}{|c|c|c|}
\hline Term & Number of pathway gene & $P$ value \\
\hline Linoleic acid metabolism & $\begin{array}{l}\text { CYP3A4, CYP2J2, CYP2C19, CYP2C9, CYP2C8, CYP2E1, CYP1A2, PLA2G4A, } \\
\text { PLA2G1B, PLA2G2A, PLA2G6, PLA2G3, PLA2G4C, PLA2G5 }\end{array}$ & $1.20 E-11$ \\
\hline VEGF signaling pathway & $\begin{array}{l}\text { PIK3CG, PRKCA, PTGS2, MAPKAPK2, PRKCB, PLA2G4A, KRAS, RAC2, MAPK14, } \\
\text { MAPK3, RAC1, PLA2G4C, AKT2 }\end{array}$ & $2.69 E-06$ \\
\hline Serotonergic synapse & $\begin{array}{l}\text { PRKCA, PLA2G4A, APP, CYP2J2, KRAS, CYP2C19, PTGS2, CYP2C9, CYP2C8, } \\
\text { CYP2D6, MAPK3, PTGS1, ALOX12B, PRKACA, ALOX5, PLA2G4C, PRKCB }\end{array}$ & $4.54 E-06$ \\
\hline Fc epsilon RI signaling pathway & $\begin{array}{c}\text { IL4, PIK3CG, PRKCA, PRKCB, PLA2G4A, KRAS, RAC2, MAPK14, MAPK3, RAC1, } \\
\text { PLA2G4C, SYK, AKT2 }\end{array}$ & $8.83 E-06$ \\
\hline Alpha-linolenic acid metabolism & ACOX1, PLA2G4A, PLA2G2A, PLA2G1B, PLA2G6, PLA2G4C, PLA2G3, PLA2G5 & $3.08 E-05$ \\
\hline Pancreatic cancer & $\begin{array}{c}\text { PIK3CG, KRAS, RAC2, TGFBR1, MAPK3, TGFBR2, RAC1, NFKB1, SMAD2, EGF, } \\
\text { TGFB1, AKT2 }\end{array}$ & $3.17 E-05$ \\
\hline $\begin{array}{l}\text { (American } \\
\text { is) }\end{array}$ & $\begin{array}{c}\text { PIK3CG, CCL3, TGFBR1, TGFBR2, NFKBIA, NFKB1, SMAD2, BDKRB2, CALR, } \\
\text { TGFB1, ACE, MAPK14, MAPK3, FAS, AKT2 }\end{array}$ & $3.98 E-05$ \\
\hline $\begin{array}{l}\text { Metabolism of xenobiotics by } \\
\text { cytochrome P } 450\end{array}$ & $\begin{array}{c}\text { CYP3A4, CYP2A13, CYP3A5, CYP1B1, CYP1A1, CYP2B6, CYP2C9, CYP2D6, } \\
\text { CYP2A6, CYP2E1, CYP1A2, AKR1C1 }\end{array}$ & $1.09 E-04$ \\
\hline Sphingolipid signaling pathway & $\begin{array}{c}\text { PRKCA, PIK3CG, SGMS2, CERS5, NFKB1, SGMS1, BDKRB2, PRKCB, KRAS, RAC2, } \\
\text { MAPK14, MAPK3, RAC1, ABCC1, AKT2 }\end{array}$ & $1.93 E-04$ \\
\hline Chemical carcinogenesis & $\begin{array}{l}\text { CYP3A4, CYP2A13, CYP3A5, CYP1B1, CYP1A1, CYP2C19, PTGS2, CYP2C9, } \\
\text { CYP2C8, CYP2A6, CYP2E1, CYP1A2 }\end{array}$ & $2.22 E-04$ \\
\hline Ras si & $\begin{array}{l}\text { PRKCA, PIK3CG, FGFR4, CSF1, NFKB1, PRKCB, PLA2G4A, KRAS, RAC2, RAC1, } \\
\text { MAPK3, PLA2G1B, PLA2G2A, PLA2G6, PRKACA, FGF1, PLA2G3, PLA2G4C, EGF, } \\
\text { PLA2G5, AKT2 }\end{array}$ & -04 \\
\hline Osteoclast differentiation & $\begin{array}{c}\text { PIK3CG, NCF2, TGFBR1, CSF1, CREB1, PPARG (PPAR } \gamma \text { P), TGFBR2, NFKBIA, } \\
\text { NFKB1, TGFB1, MAPK14, MAPK3, RAC1, AKT2, SYK }\end{array}$ & $4.83 E-04$ \\
\hline Colorectal cancer & PIK3CG, KRAS, RAC2, TGFBR1, MAPK3, TGFBR2, RAC1, SMAD2, TGFB1, AKT2 & $5.51 E-04$ \\
\hline FoxO signaling pathway & $\begin{array}{l}\text { PIK3CG, TGFBR1, TGFBR2, SMAD2, SIRT1, TGFB1, G6PC, KRAS, MAPK14, MAPK3, } \\
\text { PRKAA1, CAT, PRKAA2, EGF, AKT2 }\end{array}$ & $6.08 E-04$ \\
\hline Retinol metabolism & $\begin{array}{l}\text { CYP3A4, CYP3A5, DGAT1, CYP1A1, CYP2B6, CYP2C9, CYP2C8, HSD17B6, CYP2A6, } \\
\text { CYP1A2 }\end{array}$ & $7.00 E-04$ \\
\hline MAPK signaling pathway & $\begin{array}{l}\text { PRKCA, FGFR4, TGFBR1, TGFBR2, NFKB1, HSPA1A, HSPA1B, MAPKAPK2, TGFB1, } \\
\text { PRKCB, PLA2G4A, KRAS, RAC2, MAPK14, RAC1, MAPK3, PRKACA, FAS, FGF1, } \\
\text { PLA2G4C, EGF, AKT2 }\end{array}$ & $7.04 E-04$ \\
\hline Drug metabolism-cytochron & $\begin{array}{l}\text { CYP3A4, CYP3A5, CYP2C19, CYP2B6, CYP2C9, CYP2C8, CYP2D6, CYP2A6, } \\
\text { CYP2E1, CYP1A2 }\end{array}$ & 0.001095 \\
\hline B Cell receptor signaling pathway & PIK3CG, KRAS, RAC2, MAPK3, RAC1, CD81, NFKBIA, NFKB1, AKT2, SYK & 0.001218 \\
\hline Hepatitis C & $\begin{array}{l}\text { PIK3CG, PPARA, LDLR, RXRA, NFKBIA, NFKB1, KRAS, MAPK14, MAPK3, CD81, } \\
\text { SCARB1, EGF, AKT2, NR1H3 }\end{array}$ & 0.001755 \\
\hline Influenza A & $\begin{array}{l}\text { PRKCA, PIK3CG, IL18, FDPS, NFKBIA, NLRX1, HSPA1A, NFKB1, HSPA1B, NLRP3, } \\
\text { PRKCB, MAPK14, MAPK3, PYCARD, FAS, AKT2 }\end{array}$ & 0.002805 \\
\hline
\end{tabular}

inflammatory response and chronic low-grade inflammation [23]. Besides, kaempferol, luteolin, and quercetin can reduce the production of the proinflammatory factor IL- 6 or tumor necrosis factor (TNF) to promote the production of the anti- inflammatory factor IL-10 or reduce the expression of Cox-2 and inducible nitric oxide synthase (iNOS) to downregulate the level of NO and PGE2 [24]. Kim et al. showed that quercetin and kaempferol can significantly reduce mice's ear 
swelling, relieves writhing response, and can significantly prevent cough in mice, indicating anti-inflammatory, analgesic, and antitussive activities of these compounds [25]. In terms of antiviral effects, Yan et al. showed that luteolin has a certain inhibitory effect on influenza A virus (H1N1), as it may reduce the mRNA expression of hemagglutinin (HA) and neuraminidase (NA) in virus-infected cells [26]. In terms of antibacterial effects, extracts of luteolin and kaempferol have the highest activity against Gram-positive bacteria in vitro, especially against Streptococcus aureus, Streptococcus pneumoniae, Streptococcus epidermidis, Bacillus cereus, and Bacillus subtilis [27].

A total of 63 key targets for SXZKF in the treatment of pharyngitis were identified. DC, CC, and $\mathrm{BC}$ analysis indicated that ALB (serum albumin), PPAR $\gamma$ (peroxisome proliferator-activated receptor gamma), MAPK3 (mitogenactivated protein kinase 3), EGF (epidermal growth factor), and PTGS2 (prostaglandin G/H synthase 2) are closely related to pharyngitis. ALB is an important substance to maintain plasma colloid osmotic pressure, but bacterial infection in the throat can cause local vascular permeability to increase, lead to vasodilatation and exudation of the serous fluid, and finally result in hyperemia, pain, and even thickening of the mucosa. When localized albumin synthesis increases when the throat is inflamed, ALB acts to maintain intravascular osmotic pressure, reduce serous exudation, and alleviate the symptoms as well as processes of pharyngeal inflammation [28]. EGF promotes the growth of various epidermal cells and play an important role as a mucosal protector in mucosal defense and ulcer healing [29]. Studies have shown that EGF is related to the healing of chronic pharyngeal inflammation and participates in the pharyngeal mucosa repair [30]. PPAR $\gamma$ is an essential transcription factor, which can act as an inhibitor for inflammatory gene expression, blocking the reverses inflammation [31, 32]. MAPK can transmit signals from receptors on the cell surface to DNA in the nucleus and participate in biological processes, such as cell growth, death, and cell cycle, and also in the regulation of pathological processes, such as inflammation and stress response. As a member of the MAPK family, MAPK3 plays an important role in the process of proliferation, differentiation, and formation of inflammatory cells $[33,34]$. PTGS2 is an inducible immediate response gene that is not expressed in most cells under normal physiological conditions, but during pathological reactions such as inflammation or tumors, the expression of PTGS2 is rapidly upregulated, producing a large amount of prostaglandins. Prostaglandins are mediators of inflammation that expand blood vessels and increase the sensitivity of nerve endings to bradykinin and histamine, leading to inflammatory pain [35].

This study analyzes the six pathways that are highly relevant to the pathogenesis of pharyngitis. Vasodilation and serous exudation are important links in the pathological process of pharyngitis. Vascular endothelial growth factor (VEGF) is the most effective angiogenesis factor in the body, which can bind to specific receptors of vascular endothelial cells, promote the division and proliferation of vascular endothelial cells, and promote the generation of new blood vessels and increase vascular permeability [36]. VEGFmediated signaling pathways can regulate the proliferation, migration, and survival of vascular endothelium cell, which cause changes in vascular permeability, leading to vasodilation, exudation, and inflammation. Study had shown that serum VEGF in pharyngitis model rats is higher than normal rats and decreases after treatment with TCM [37]. Serotonin, also known as 5-HT, is a nerve-conducting substance. Studies have demonstrated that 5-HT can increase the permeability of submucosal blood vessels, promote plasma extravasation, and increase congestion and edema, causing sore throat [20]. Serotonergic synaptic pathways can regulate the binding of serotonin to mediated receptors and alleviate pain symptoms. The etiology of some chronic pharyngitis is related to allergic reactions, and subjective symptoms of patients include pharyngeal foreign body sensation, itchy throat, pharynx swelling, and dry cough symptoms [38]. The combination of immunoglobulin E(IgE) with FceRI and Fc epsilon RI signal transduction pathway are the keys in causing allergic diseases [39]. The Fc epsilon RI signal transduction pathway can regulate the process of allergy-associated pharyngitis. The Ras signaling pathway is mainly composed of signal pathways such as MAPK and PI3K-AKt (phosphatidylinositol-3-kinase-protein kinase B), which can regulate and direct the differentiation of $\mathrm{CD} 4+\mathrm{T}$ lymphocytes, thereby reducing the inflammatory response. The MAPK pathway plays an important role in regulating the inflammatory response, and its function mainly includes three cascades mediated by ERK1/2, JNK, and p38 MAPK. Inactivating this pathway can reduce inflammatory cytokine production and relieve the inflammatory response [40]. Last but not least, as previously explained, influenza virus is an important factor in causing pharyngitis, while SXZKF can play an antiviral role in the treatment of pharyngitis by regulating the influenza A signaling pathway.

\section{Conclusion}

In conclusion, this study identified the main bioactive ingredients, core target genes and molecular mechanisms of SXZKF in the treatment of pharyngitis through network pharmacology. A total of 102 bioactive compounds were found, with arachidonic acid, quercetin, kaempferol, eicosapentaenoic acid, and luteolin identified as main active compounds. Among them, 63 key target genes were identified, with ALB, PPAR $\gamma$, MAPK3, EGF, and PTGS2 recognized as core targets. The main molecular mechanisms of SXZKF for pharyngitis consisted of 28 signaling pathways, and the key pathways that were closely related to pharyngitis were found to be related to analgesia, inhibition of inflammation response, inhibition of viral replication, and inhibition of anaphylactic reaction through inactivating serotonergic synaptic, VEGF signaling pathway, Fc epsilon RI signaling pathway, Ras signaling pathway, MAPK signaling pathway, and influenza A. Taken together, this study provides an insight on the cellular and pathway mechanism of SXZKF in the treatment of pharyngitis. This new technique could also be used to understand many other 
traditional or alternative medicine to provide a new horizon for the development of personalized TCM in the near future.

$\begin{array}{ll}\text { Abbreviations } \\ \text { SXZKF: } & \text { Sang-Xing-Zhi-Ke-Fang } \\ \text { TCMSP: } & \text { Traditional Chinese Medicine Systems } \\ & \text { Pharmacology Database and Analysis } \\ & \text { Platform } \\ \text { BATMAN- } & \text { Bioinformatics Analysis Tool for Molecular } \\ \text { TCM: } & \text { mechANism of Traditional Chinese Medicine } \\ \text { UniProt: } & \text { Universal Protein Database } \\ \text { CTD: } & \text { Comparative Toxicogenomics Database } \\ \text { GeneCards: } & \text { Human Gene Database } \\ \text { STRING: } & \text { Search Tool for the Retrieval of Interacting } \\ & \text { Genes } \\ \text { PPI: } & \text { Protein-protein interactions } \\ \text { DC: } & \text { Degree centrality } \\ \text { BC: } & \text { Betweenness centrality } \\ \text { CC: } & \text { Closeness centrality } \\ \text { DAVID: } & \text { Database for Annotation, Visualization, and } \\ \text { KEGG: } & \text { Integrated Discovery } \\ \text { OB: } & \text { Kyoto Encyclopedia of Genes and Genomes } \\ \text { DL: } & \text { Oral bioavailability } \\ \text { ALB: } & \text { Drug-likeness } \\ \text { PPAR } \gamma: & \text { Serum albumin } \\ \text { PAPK3: } & \begin{array}{l}\text { Peroxisome proliferator-activated receptor } \\ \text { gamma }\end{array} \\ \text { Mitogen-activated protein kinase 3 } & \text { Epidermal growth factor } \\ \text { PI3K-AKt: } & \text { Prostaglandin G/H synthase 2 } \\ & \text { Phosphatidylinositol-3-kinase-protein } \\ \text { kinase B. }\end{array}$

\section{Data Availability}

The data used in this study are available at the following links: TCMSP: http://lsp.nwu.edu.cn/tcmsp.php; BATMANTCM: http://bionet.ncpsb.org/batman-tcm/; CTD: http:// ctdbase.org/; GeneCards: http://www.genecards.org/; Draw Venn diagrams: http://bioinformatics.psb.ugent.be/webtools/ Venn/;STRING database: https://string-db.org/; DAVID ver 6.8: https://david.ncifcrf.gov/;OmicShare tools: https://www. omicshare.com/tools.

\section{Conflicts of Interest}

The authors declare that they have no conflicts of interest.

\section{Authors' Contributions}

Liu Qiong proposed the idea and designed this study. Yinhe Deng carried out all experiments required in the study. Quanjiang Li participated in data analysis, and Menglin Li and Tiantian Han wrote and improved the manuscript. Guixian Li carried out literature survey. All authors read and approved the final manuscript.

\section{Acknowledgments}

This work was funded by Science and Technology Agency of Guangdong Province (201904010235) and The First Affiliated Hospital of Guangzhou University of Chinese Medicine (2019ZJ06).

\section{Supplementary Materials}

Table S1: 102 bioactive compounds obtained and screened out from TCMSP, BATMAN-TCM, and literature. Table S2: 886 targets of bioactive compounds collected using TCMSP and UniProt. Table S3: targets related to pharyngitis, including 5150 targets from CTD and 1803 targets from GeneCards with 695 targets duplicated. Table S4: 387 overlapping targets related to 19 bioactive compounds. Table S5: the results of topological features of the PPI network, including the values of topological features of 354 targets, while the other 33 targets were unrelated to each other target in the network. Table S6: the results of KEGG pathway enrichment, including 43 KEGG pathways were recognized as $P<0.05$ with 28 pathways being recognized as $P<0.01$. (Supplementary Materials)

\section{References}

[1] S. Wu, X. Peng, Z. Yang et al., "Estimated burden of group a streptococcal pharyngitis among children in Beijing, China," BMC Infectious Diseases, vol. 16, no. 1, p. 452, 2016.

[2] H. L. Jiang, B. She, W. Liu, B. Mao, and J. Y. Zhang, "Efficacy and safety of Qi-Wei-Qing-Yan aerosol in treatment of acute pharyngitis (lung-stomach excess-heat syndrome): study protocol for a randomized controlled trial," Trials, vol. 17, no. 1, p. 99, 2016.

[3] X. Y. Yao, Y. J. Bian, Y. Gao et al., "Clinical application evaluation and revision suggestions of clinical practice guideline on traditional Chinese medicine therapy alone or combined with antibiotics for acute pharyngitis," Zhongguo Zhong Yao Za Zhi=Zhongguo Zhongyao Zazhi=China Journal of Chinese Materia Medica, vol. 43, no. 24, pp. 4765-4770, 2018.

[4] C. X. Li, F. C. Wu, W. L. Yuan et al., "Systematic review of herbal tea (a traditional Chinese treatment method) in the therapy of chronic simple pharyngitis and preliminary exploration about its medication rules," Evidence-Based Complementary and Alternative Medicine, vol. 2019, Article ID 9458676, 2019.

[5] C. Lu, Y. Song, J. Zhang et al., "Yanshu spraying agent, a traditional Chinese medicine, relieves chronic pharyngitis in animals by anti-inflammatory and antibacterial effects," $E x$ perimental and Therapeutic Medicine, vol. 7, no. 4, pp. 990994, 2014

[6] Q. L. Du, A Clinical Observation of Varied Sangxing Decotion for Wind Dry Lung Injury Syndrome of Post-Infectious Cough, Guangzhou University of Chinese Medicine, Guangzhou, China, 2017.

[7] S. Y. Chan and J. Loscalzo, "The emerging paradigm of network medicine in the study of human disease," Circulation Research, vol. 111, no. 3, pp. 359-374, 2012.

[8] Z. Liu, F. Guo, Y. Wang et al., "BATMAN-TCM: a bioinformatics analysis tool for molecular mechANism of 
traditional Chinese medicine," Scientific Reports, vol. 6, Article ID 21146, 2016.

[9] S. J. Huang, F. Mu, F. Li et al., "Systematic elucidation of the potential mechanism of erzhi pill against drug-induced liver injury via network pharmacology approach," Evidence Based Complementary Alternative Medicine, vol. 2020, Article ID 6219432, 2020.

[10] T. H. Liu, W. H. Chen, X. D. Chen et al., "Network pharmacology identifies the mechanisms of action of TaohongSiwu decoction against essential hypertension," Medical Science Monitor: International Medical Journal of Experimental and Clinical Research, vol. 26, Article ID e920682, 2020.

[11] X. C. Zhang, "Research progress of pharmacology and clinical effects of pheretima," Chinese Traditional Patent Medicine, in China, vol. 33, no. 9, pp. 1574-1578, 2011.

[12] Q. Huang, Z. W. Li, Z. G. Ma et al., "Research progress of pheretima," Chinese Journal of Experimental Traditional Medical Formulae, in China, vol. 24, no. 13, pp. 220-226, 2018.

[13] E. Boutet, D. Lieberherr, M. Tognolli et al., "UniProtKB/ Swiss-Prot, the manually annotated section of the UniProt KnowledgeBase: how to use the entry view," Methods in Molecular Biology (Clifton, N.J.), vol. 1374, pp. 23-54, 2016.

[14] S. Pundir, M. J. Martin, and C. O’Donovan, "UniProt protein knowledgebase," Methods in Molecular Biology (Clifton, N.J.), vol. 1558, pp. 41-55, 2017.

[15] A. P. Davis, C. J. Grondin, R. J. Johnson et al., "The comparative toxicogenomics database: update 2019," Nucleic Acids Research, vol. 47, no. D1, pp. D948-D954, 2019.

[16] Y. Tang, M. Li, J. Wang et al., "CytoNCA: a cytoscape plugin for centrality analysis and evaluation of protein interaction networks," Bio Systems, vol. 127, pp. 67-72, 2015.

[17] X. Du, L. Zhao, Y. Yang et al., "Investigation of the mechanism of action of Porana sinensis Hemsl. against gout arthritis using network pharmacology and experimental validation," Journal of Ethnopharmacology, vol. 252, Article ID 112606, 2020.

[18] A. M. Harris, L. A. Hicks, and A. Qaseem, “Appropriate antibiotic use for acute respiratory tract infection in adults: advice for high-value care from the American college of physicians and the centers for disease control and prevention," Annals of Internal Medicine, vol. 164, no. 6, pp. 425-434, 2016.

[19] Q. B. Chen, Y. Wang, and F. Li, "Research progress on etiology and pathogenesis of chronic pharyngitis," Journal of Audiology and Speech Pathology, in China, vol. 27, no. 2, pp. 224-228, 2019.

[20] J. B. Wu, D. F. Huang, Y. Wang et al., "Overview on animal models of throat impediment," Chinese Journal of Experimental Traditional Medical Formulae, in China, vol. 25, no. 18, pp. 189-195, 2019.

[21] Y. Zhang, H. Chen, W. Zhang et al., "Arachidonic acid inhibits inflammatory responses by binding to myeloid differentiation factor-2 (MD2) and preventing MD2/toll-like receptor 4 signaling activation," Biochimica et Biophysica Acta-Molecular Basis of Disease, vol. 1866, no. 5, Article ID 165683, 2020.

[22] M. Murata, H. Kaji, Y. Takahashi et al., "Stimulation by eicosapentaenoic acids of leptin mRNA expression and its secretion in mouse 3T3-L1 adipocytes in vitro," Biochemical and Biophysical Research Communications, vol. 270, no. 2, pp. 343-348, 2000.
[23] P. C. Calder and R. F. Grimble, "Polyunsaturated fatty acids, inflammation and immunity," European Journal of Clinical Nutrition, vol. 56, no. Suppl 3, pp. S14-S19, 2002.

[24] M. Mueller, S. Hobiger, and A. Jungbauer, "Anti-inflammatory activity of extracts from fruits, herbs and spices," Food Chemistry, vol. 122, no. 4, pp. 987-996, 2010.

[25] J. X. Zhu, L. Wen, W. J. Zhong, L. Xiong, J. Liang, and H.-L. Wang, "Quercetin, kaempferol and isorhamnetin in elaeagnus pungens thunb. leaf: pharmacological activities and quantitative determination studies," Chemistry \& Biodiversity, vol. 15, no. 8, Article ID e1800129, 2018.

[26] Y. Haiyan, M. Linlin, W. Huiqiang et al., "Luteolin decreases the yield of influenza A virus in vitro by interfering with the coat protein I complex expression," Journal of Natural Medicines, vol. 73, no. 3, pp. 487-496, 2019.

[27] M. Kozyra, A. Biernasiuk, A. Malm, and M. Chowaniec, "Chemical compositions and antibacterial activity of extracts obtained from the inflorescences of Cirsium canum (L.) all," Natural Product Research, vol. 29, no. 21, pp. 2059-2063, 2015.

[28] L. Happonen, S. Hauri, G. Svensson Birkedal et al., "A quantitative Streptococcus pyogenes-human protein-protein interaction map reveals localization of opsonizing antibodies," Nature Communications, vol. 10, no. 1, p. 2727, 2019.

[29] J. A. Clark, R. H. Lane, N. K. Maclennan et al., "Epidermal growth factor reduces intestinal apoptosis in an experimental model of necrotizing enterocolitis," American Journal of physiology. Gastrointestinal and Liver Physiology, vol. 288, no. 4, pp. G755-G762, 2005.

[30] X. J. Wu, B. Z. Su, L. Z. Wu et al., "Curative efficacy of qingkailing dripping pills on chronic pharyngitis and its effects on serum EGF IL-1 beta and VCAM-1," Journal of Hebei Medicine, in China, vol. 24, no. 12, pp. 1960-1965, 2018.

[31] S. Muralikumar, U. Vetrivel, A. Narayanasamy, and U. N. Das, "Probing the intermolecular interactions of PPARgamma-LBD with polyunsaturated fatty acids and their anti-inflammatory metabolites to infer most potential binding moieties," Lipids in Health and Disease, vol. 16, no. 1, p. 17, 2017.

[32] H. S. Cheng, W. R. Tan, Z. S. Low et al., "Exploration and development of PPAR modulators in health and disease: an update of clinical evidence," International Journal of Molecular Sciences, vol. 20, no. 20, 2019.

[33] J. Liu, L. Liu, Y. Cui, J. Zhang, and H. Jiang, "p38 MAPK regulates Th2 cytokines release in PBMCs in allergic rhinitis rats," Journal of Huazhong University of Science and Technology. Medical sciences = Hua Zhong Ke Ji Da Xue Xue Bao. Yi xue Ying De wen Ban=Huazhong Keji Daxue Xue Bao. Yixue Yingdewen Ban, vol. 30, no. 2, pp. 222-225, 2010.

[34] H. I. Kim, S. H. Hong, J. M. Ku et al., "Tonggyu-tang, a traditional Korean medicine, suppresses pro-inflammatory cytokine production through inhibition of MAPK and NF-kappaB activation in human mast cells and keratinocytes," BMC Complementary and Alternative Medicine, vol. 17, no. 1, p. 186, 2017.

[35] D. F. Mansour, D. O. Saleh, and R. E. Mostafa, "Genistein ameliorates cyclophosphamide-induced hepatotoxicity by modulation of oxidative stress and inflammatory mediators," Open Access Macedonian Journal of Medical Sciences, vol. 5, no. 7, pp. 836-843, 2017.

[36] W. Zha, M. Su, M. Huang, J. Cai, and Q. Du, “Administration of pigment epithelium-derived factor inhibits airway inflammation and remodeling in chronic OVA-induced mice via VEGF suppression," Allergy, Asthma \& Immunology Research, vol. 8, no. 2, pp. 161-169, 2016. 
[37] L. Kai, N. Le, J. Lili et al., "Effects of different decoction methods of Huanglian Ejiao decoction on rat model with chronic pharyngitis China," Journal of Traditional Chinese Medicine and Pharmacy, in China, vol. 29, no. 3, pp. 879-881, 2014.

[38] J. P. Wu, Z. D. Mei, Z. Z. Tao et al., "Diagnosis and treatment of allergic pharyngitis," Journal of Clinical Otorhinolarynglolgy Head and Neck Surgery, in China, vol. 22, p. 1047, 2006.

[39] Z. C. Liu, H. L. Shi, Y. F. Zhang et al., "Progress in the study of allergic disease drugs targeting on IgE/FceRI signaling pathway," Acta Pharmaceutica Sinica, in China, vol. 46, no. 10, pp. 1161-1166, 2011.

[40] C. Gefu, W. Miaomiao, X. Xianxing, L. W. Soromou, F. Liu, and S. Zhao, "Suppression of MAPK and NF- $\kappa$ B pathways by limonene contributes to attenuation of lipopolysaccharideinduced inflammatory responses in acute lung injury," Inflammation, vol. 36, no. 2, pp. 501-511, 2013. 